\title{
Torque Limit-Based Inertial Control of a DFIG for Rapid Frequency Stabilization
}

\author{
Yien Xu and Hongmei Wang* \\ Department of Electrical Engineering, Nantong University, Nantong, China
}

\section{OPEN ACCESS}

Edited by:

Liansong Xiong,

Nanjing Institute of Technology (NUIT),

China

Reviewed by:

Jun Cong Ge,

Jeonbuk National University, South Korea

Huimin Wang,

University of Electronic Science and

Technology of China, China

Zhaoyang Jin,

Shandong University, China

*Correspondence:

Hongmei Wang

wanghongmei@ntu.edu.cn

Specialty section:

This article was submitted to

Process and Energy Systems

Engineering,

a section of the journal

Frontiers in Energy Research

Received: 04 October 2021

Accepted: 21 October 2021

Published: 22 November 2021

Citation:

Xu Y and Wang H (2021) Torque Limit-

Based Inertial Control of a DFIG for

Rapid Frequency Stabilization.

Front. Energy Res. 9:788989.

doi: 10.3389/fenrg.2021.788989
With the increasing penetration of renewable energy generation, the frequency stability of a power grid can be significantly threatened. A doubly-fed induction generator (DFIG) participates in the frequency support of a power grid by releasing kinetic energy (KE) to boost the frequency nadir (FN). However, during rotor speed restoration, it is difficult to counterbalance the size of a second frequency drop (SFD) and the rotor speed recovery duration. This paper proposes an improved torque limit-based inertial control (TLBIC) to raise the FN by releasing less kinetic energy while guaranteeing rapid frequency stabilization with reduced SFD. To this end, when detecting a disturbance, the DFIG enhances the active reference power to the torque limit, and then the active power reduces smoothly based on an exponential function until the maximum power point tracking (MPPT) curve is met, and the rotor speed reverts to the initialization operating condition along the MPPT curve. A simulation system model with various wind power penetrations is established in EMTP-RV. Results show that the proposed scheme boosts the $\mathrm{FN}$ at a high level with less $\mathrm{KE}$ and guarantees rapid frequency stabilization.

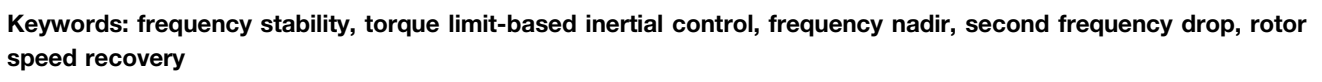
speed recovery

\section{INTRODUCTION}

Recently, wind power generation has developed rapidly due to the shortage of fossil fuel and worsening environmental situations. Doubly-fed induction generators (DFIGs) are widely applied in the field of wind power because of the relatively low price, decoupling control operation of the active power and reactive power, maximum power point tracking (MPPT) control, deloading operation, and other advanced control strategies (Bao et al., 2020; Xiong et al., 2020). In addition, DFIGs are connected to the grid by power electronic converters, which decouple the rotor speed of the wind turbine from the system frequency. Consequently, DFIGs are unable to sustain the system frequency (Yang et al., 2022; Yang et al., 2021; Xiong et al., 2021a). With the increasing penetration level of wind power, the power system will face significant challenges in system frequency stability. Therefore, more and more attention has been paid to DFIG active frequency support (Wang and Tomsovic, 2018; Xiong et al., 2021b).

DFIGs supply inertial control using additional control strategies. In Kheshti et al. (2019), the most characteristic inertial control can be roughly divided into two types: virtual inertial control (PD control) (Morren et al., 2006; Fernandez et al., 2008; Lee et al., 2016; Van de Vyver et al., 2016; Li et al., 2017; Hu and Wu, 2019; Peng et al., 2020) and stepwise inertial control (Ullah et al., 2008; Lao, 2021). PD control emulates the inertia response and primary frequency 
response of synchronous generations (SGs) to provide a frequency response. Compared with PD control, stepwise inertial control can supply a rapid frequency response due to the operating characteristics of a DFIG (Kheshti et al., 2019). However, after DFIGs participate in frequency regulation by releasing rotor kinetic energy, the rotor speed will deviate from the optimal state corresponding to MPPT operation. Therefore, the rotor speed needs to regain the optimal state considering the economic benefits of the wind farm, but the speed recovery is accompanied by power mutation, which trend to cause a significant SFD (Xiong et al., 2021c).

In Hafiz and Abdennour (2015), the active power is designed as a slope function. Even though this scheme lessens the SFD to a certain extent, but it delays the rotor speed recovery. Furthermore, the parameters of this function in different scenarios need to be reset. In order to recover the rotor speed, a constant power reference is addressed (Kang et al., 2016a; Kang et al., 2016b). However, the tradeoff between the depth of an SFD and the rotor speed restoration is difficult to achieve. The study of $\mathrm{Xu}$ and $\mathrm{Xu}$ (2017) suggests a constant accelerating power reference based on the mechanical power curve, which realizes the smooth recovery of the rotor speed. Nevertheless, the mechanical power curve is difficult to acquire and SFD still exists due to the sudden power drop. The authors of (Lao, 2021) suggested a frequency regulation strategy based on logistic regression function, which realizes smooth rotor speed recovery. However, the function used in this strategy is too complex, and too many parameters need to be defined, which brings difficulties to engineering applications. To compensate for the energy required for rotor speed recovery, a coordinated frequency control strategy for the wind turbine and SG is addressed ( $\mathrm{Xu}$ et al., 2018). The rotor speed recovery can be realized with reduced SFD by adjusting the primary frequency modulation parameters of the SG. However, for a large-scale power system with high wind power penetrations, this strategy is not economical to regain the rotor speed. In Wu et al. (2017), a frequency control strategy based on the energy storage system is suggested, which realizes the rapid speed recovery while eliminating the SFD. However, the overall operation and maintenance cost of the power system increases due to the high cost of the energy storage system.

To approach the above issues, this paper addresses an improved torque limit-based inertial control (TLBIC) scheme to raise the $\mathrm{FN}$ by releasing less rotational energy while guaranteeing rapid frequency stabilization with reduced SFD. To this end, when detecting a disturbance, the power reference of the DFIG increases to the torque limit. Afterward, the power reference decreases smoothly based on an exponential function until it the maximum power point tracking (MPPT) curve is met, and then the rotor speed reverts to the initialization operating condition along the MPPT curve. In addition, DFIGs are assumed to work in MPPT mode prior to frequency disturbance. The benefits of the proposed TLBIC scheme are verified under various wind power penetrations and wind speed conditions based on an EMTP-RV simulator.

\section{MODELING OF A DOUBLY-FED INDUCTION GENERATOR}

This section mainly introduces the typical structure of a DFIG, as illustrated in Figure 1 which includes a wind turbine model, twomass shaft model, and DFIG controllers.

\section{Wind Turbine Model}

The mechanical input power captured by a wind turbine from moving air is represented as:

$$
P_{m}=\frac{1}{2} c_{P}(\lambda, \beta) \rho A v_{w}^{3}=\frac{1}{2} c_{P}(\lambda, \beta) \rho \pi R^{2} v_{w}^{3}
$$

where $c_{p}$ represents the power coefficient; $\lambda$ and $\beta$ are the tipspeed ratio and pitch angle, respectively; $\rho$ is the air density; $A$ indicates the swept area by blade; $R$ means the blade length and $v_{w}$ means the wind velocity.

As in (Kang et al., 2016a; Kang et al., 2016b), $c_{p}$ employed in Eq. 1 can be represented as:

$$
c_{P}(\lambda, \beta)=0.645\left\{0.00912 \lambda+\frac{-5-0.4(2.5+\beta)+116 \lambda_{i}}{e^{21 \lambda_{i}}}\right\}
$$

where

$$
\lambda_{i}=\frac{1}{\lambda+0.08(2.5+\beta)}-\frac{0.035}{1+(2.5+\beta)^{3}}
$$

and $\lambda$ is given as:

$$
\lambda=\frac{\omega_{r} R}{v_{w}}
$$

where $\omega_{r}$ represents the rotor speed of the generator.

For capturing more wind energy resources, the DFIG usually works in MPPT operation. As in Fernandez et al. (2008), the MPPT operation power reference, $P_{M P P T}$, is expressed as:

$$
P_{M P P T}=\frac{1}{2} c_{P, \max } \rho \pi R^{2}\left(\frac{\omega_{r} R}{\lambda_{\text {opt }}}\right)^{3}=k \omega_{r}^{3}
$$

where $c_{p, \max }$ is a maximum value of $c_{p}$ when $\beta=0^{\circ}, \lambda=\lambda_{\text {opt }}$ and set to 0.5 in this paper; $\lambda_{o p t}$ is the optimal $\lambda$ of the wind turbine to capture the maximum wind energy and set to 9.95 in this paper; $k$ is a calculation of the characteristic parameters of the wind turbine and set to 0.512 in this paper.

\section{Two-Mass Shaft Model}

This paper uses a two-mass shaft model to express the mechanical dynamics between the wind turbine and induction generator, the model can be represented as Eqs 6-9 in (Boukhezzar and Siguerdidjane, 2011).

$$
\begin{aligned}
J_{t} \frac{d \omega_{t}}{d t} & =T_{m}-T_{L} \\
J_{g} \frac{d \omega_{r}}{d t} & =T_{H}-T_{e m} \\
T_{L} & =K \theta+D\left(\omega_{t}-\omega_{L}\right)
\end{aligned}
$$




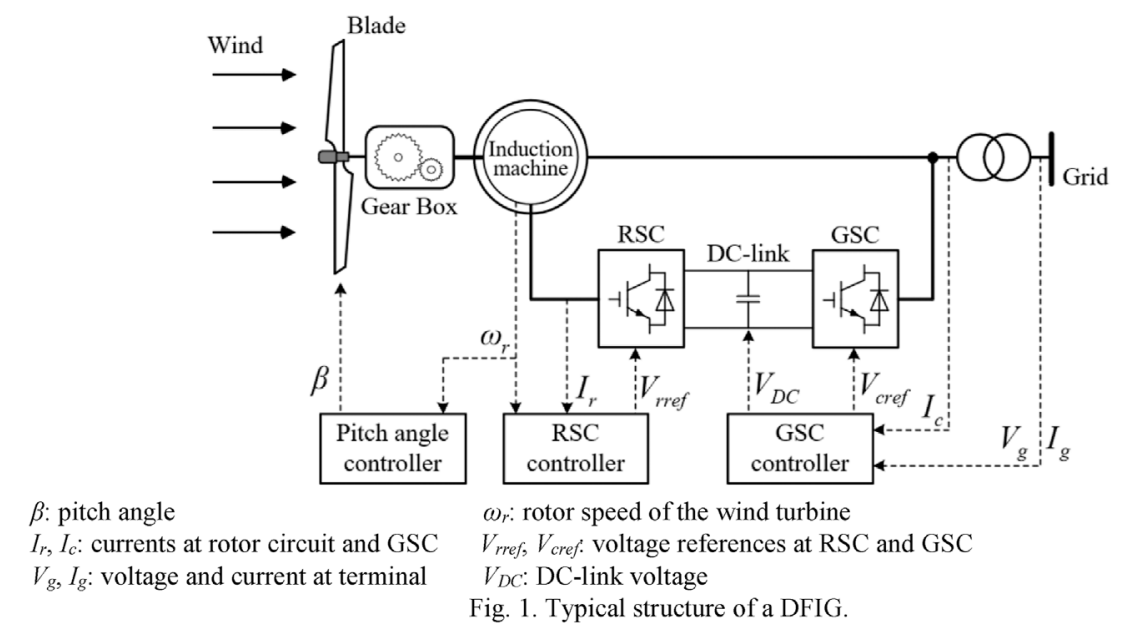

FIGURE 1 | Typical structure of a DFIG.

$$
N=\frac{T_{L}}{T_{H}}=\frac{\omega_{r}}{\omega_{L}}
$$

where $J_{\mathrm{t}}$ and $J_{\mathrm{g}}$ are the inertia constants of the wind turbine and generator, respectively; $T_{m}$ and $T_{e m}$ are the mechanical torque, electrical torque of the wind turbine and generator, respectively; $\omega_{t}$ is the rotor speed of the wind turbine; $T_{L}$ and $T_{H}$ are the torques of the low-speed and the high-speed shafts, respectively; $\omega_{L}$ is the rotor speed of the low-speed shaft; $K$ is the spring constant; $D$ is the damping constant; $\theta$ is the torsional twist; $N$ is the gear ratio.

$\beta$ : pitch angle $\omega_{r}$ : rotor speed of the wind turbine.

$I_{r}, I_{c}$ : currents at rotor circuit and GSC $V_{\text {rref }}, V_{\text {cref }}$ : voltage references at RSC and GSC.

$V_{g}, I_{g}$ : voltage and current at terminal $V_{D C}$ : DC-link voltage.

\section{Control System of a DFIG}

The control system consists of a pitch angle controller, a rotorside controller (RSC) and grid-side controller (GSC). The pitch angle controller adjusts the pitch angle according to the current wind speed to ensure the maximum of DFIG output power on the premise of the stable operation. An RSC realizes active and reactive power decoupling control and MPPT control of the DFIG. The GSC is mainly responsible for maintaining the DC-link voltage.

When the wind turbine output power is abnormally excessive or rapidly increased, the rapid change of the torque is inevitable. Especially in severe cases, severer mechanical torsion may damage the wind turbine. In order to protect the mechanical structure of the wind turbine and ensure the safe operation, the output power should meet Kang et al. (2016a)

$$
P_{e m} \leq P_{T \lim }=T_{\lim } \omega_{r}
$$

where $P_{e m}$ is the electromagnetic output power; $P_{\text {Tlim }}$ is the maximum reference power based on torque limit; $T_{\text {lim }}$ indicates the torque limit of the wind turbine and set to 0.88 p. u. in this paper.

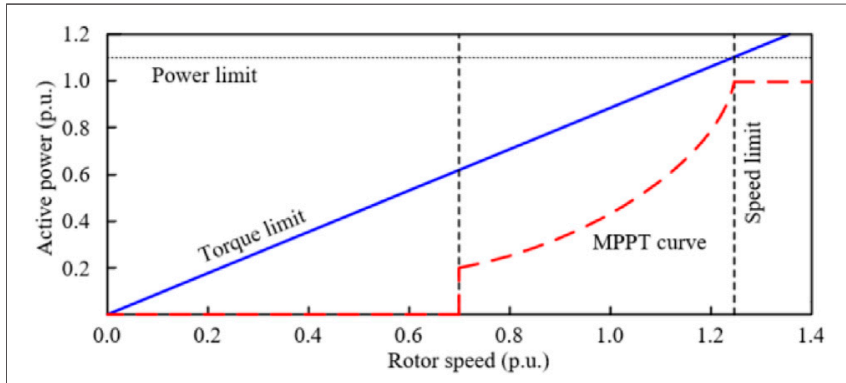

FIGURE 2 | Power characteristic curve of a DFIG.

Furthermore, the maximum power is $1.10 \mathrm{p}$. u., the minimum rotor speed is $0.70 \mathrm{p}$. u., and the maximum rotor speed is $1.25 \mathrm{p}$. u. as in Yang et al. (2018) (Figure 2).

\section{ANALYSIS THE INERTIAL CONTROL OF A DFIG ON THE DYNAMIC SYSTEM FREQUENCY}

When the DFIG implements the inertial control, the relationship between the active power variation $\left(\Delta P_{I C}\right)$ and the rotor speed of the DFIG can be represented as:

$$
\Delta P_{I C}=J_{D F I G} \omega_{r} d \omega_{r} / d t
$$

where $J_{D F I G}$ means the moment of inertia of the DFIG.

According to the definition of the inertia constant of the SG, the inertia constant of the DFIG $\left(H_{D F I G}\right)$ can be expressed as:

$$
H_{D F I G}=J_{D F I G} \omega_{n}^{2} / 2 S_{D F I G}
$$

where $\omega_{n}$ and $S_{D F I G}$ indicate the rated rotor speed and rated capacity of the DFIG, respectively. 


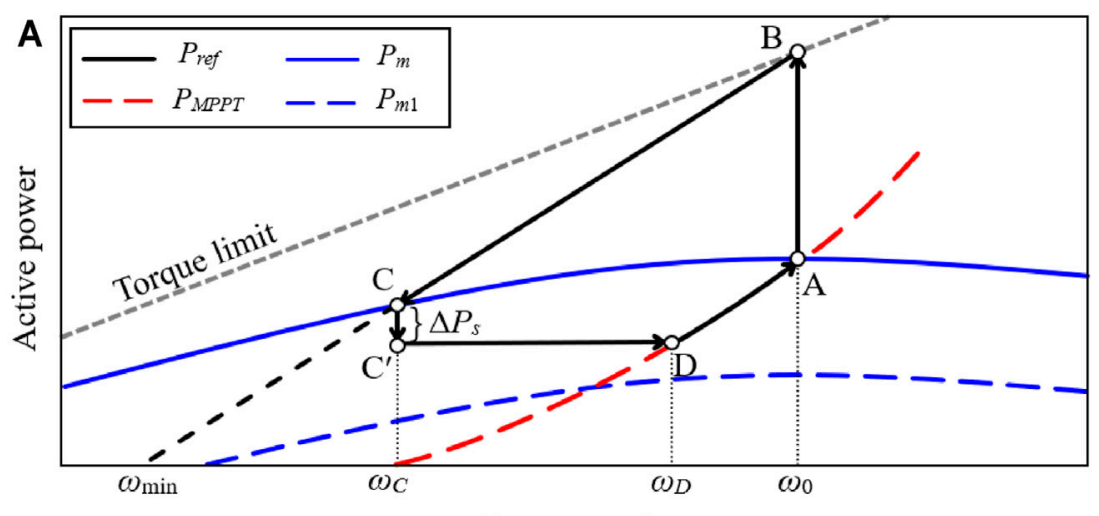

Rotor speed

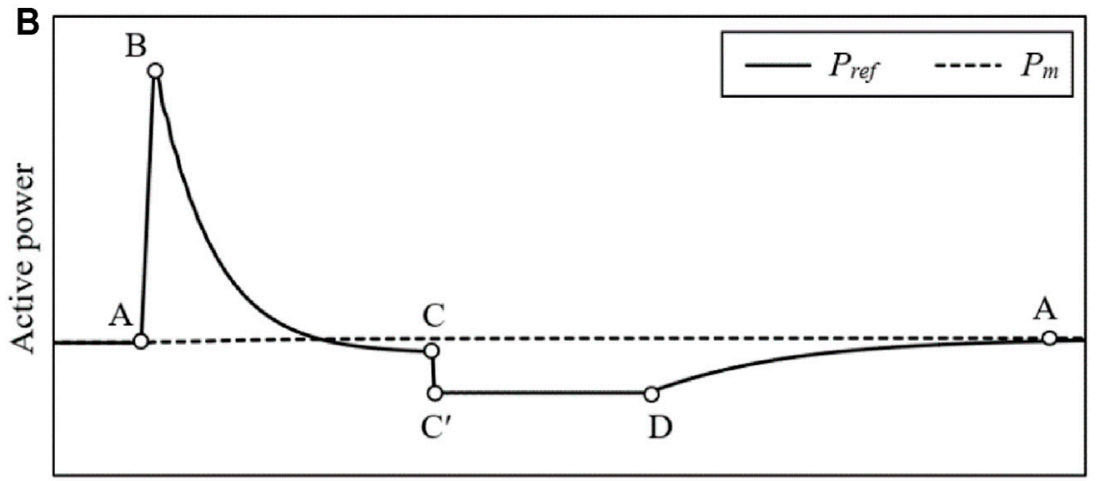

Time

FIGURE 3 | Operational features of the conventional TLBIC scheme: (A) Power trajectory in the $\omega_{r}$ domain; (B) Active power in the time domain.

Rearranging Eq. 11 and converting it to a per unit (p.u.) system, the expression of Eq. 11 can be modified as in Eq. 13

$$
\Delta P_{I C_{-} p u}=2 H_{D F I G} \omega_{p u} \frac{d \omega_{p u}}{d t}
$$

where $\Delta P_{I C_{-} p u}$ and $\omega_{p u}$ are $\Delta P_{I C}$ and the rotor speed of the DFIG in p. u., respectively.

By integrating both sides of Eqs 13, 14 is obtained

$$
\Delta E_{p u}=H_{D F I G}\left[\omega_{p u}^{2}\left(t_{1}\right)-\omega_{p u}^{2}\left(t_{0}\right)\right]
$$

where $\Delta E_{p u}$ is the released kinetic energy of the DFIG in p. u. during the inertia response, $\omega_{p u}\left(t_{1}\right)$ and $\omega_{p u}\left(t_{0}\right)$ are the rotor speed of the DFIG at $t_{1}$ and $t_{0}$ in p. u., respectively.

As in Miao et al. (2015), since the system frequency in p. u. is the same as $\omega_{p u}, \omega_{p u}$ in Eq. 14 is replaced by the frequency in p. u., thus, the expression of Eq. 14 can be modified as:

$$
f_{p u}\left(t_{1}\right)=\sqrt{\frac{\Delta E_{p u}}{H_{D F I G}}+f_{p u}^{2}\left(t_{0}\right)}
$$

where $f_{p u}\left(t_{1}\right)$ and $f_{p u}\left(t_{0}\right)$ are the system frequency at $t_{1}$ and $t_{0}$ in p. u., respectively.
Therefore, the frequency deviation, $\Delta f_{p u}=f_{p u}\left(t_{1}\right)-f_{p u}\left(t_{0}\right)$, is given by Eq. 16:

$$
\Delta f_{p u}=-f_{p u}\left(t_{0}\right)+\sqrt{\frac{\Delta E_{p u}}{H_{D F I G}}+f_{p u}^{2}\left(t_{0}\right)}
$$

As shown in Eq. 16, it is evidenced that the DFIG can support the system frequency by inertia response. The large $\Delta E_{p u}$ can reduce the system frequency deviation so as to boost the FN.

\section{TORQUE LIMIT-BASED INERTIAL CONTROL OF A DFIG}

After the KE stored in rotor is released to support the system frequency, the rotor speed will deviate from the initial operation state, and even cause the wind turbine stalling. Furthermore, the rotor speed recovery trends to cause a significant SFD due to the reduction on the output power. In order to effectively heighten the $\mathrm{FN}$ without a stalling and recover the rotor speed with reduced SFD, the inertial control strategy of the DFIG should be carefully designed.

The following section briefly introduces the characteristics of the conventional TLBIC scheme of (Kang et al., 2016a). 
Thereafter, the proposed TLBIC scheme is described in detailed in the second part of this section.

\section{Conventional Torque Limit-based Inertial Control Scheme of a DFIG.}

To improve the FN without causing the wind turbine stalling, the process of the TLBIC contains two periods: period of supporting the system frequency (A-B-C trajectory) and period of recovering rotor speed of the wind turbine (C-C'-D-A trajectory)

(Figure 3A).

\section{Period of Supporting the System Frequency}

As shown in A-B-C trajectory of Figure 3A, the DFIG operates at MPPT mode prior to a disturbance, which corresponds to Point A. Upon detecting a disturbance, the power reference of a DFIG instantly increases to Point B (which is the torque limit) along Line A-B so as to boost the FN effectively. To avoid the wind turbine stalling, the reference power $P_{\text {ref }}$ decreases along Point B to Point C. During the period of supporting the system frequency, the reference power $P_{r e f}$, is given by:

$$
P_{r e f}=P_{M P P T}\left(\omega_{\min }\right)+\frac{P_{T \lim }\left(\omega_{0}\right)-P_{M P P T}\left(\omega_{\min }\right)}{\omega_{0}-\omega_{\min }}\left(\omega_{r}-\omega_{\min }\right)
$$

where $\omega_{\min }$ is the minimum rotor speed; $\omega_{0}$ is the rotor speed prior to a disturbance; $P_{M P P T}\left(\omega_{\min }\right)$ and $P_{M P P T}\left(\omega_{0}\right)$ are the value of $P_{M P P T}$ at $\omega_{\min }$ and $\omega_{0}$, respectively; $P_{\text {Tlim }}\left(\omega_{0}\right)$ is the maximum power corresponding to torque limit at $\omega_{0}$.

Since $P_{r e f}$ is greater than the input mechanical power $P_{m}, \omega_{r}$ decreases continuously based on the swing equation. Besides, as described in Eq. 17, the power reference $P_{r e f}$ is a linear function of $\omega_{r}$, the active power output will decrease along the Line B-C with $\omega_{r}$ (see B-C trajectory in Figure 3A). When $P_{\text {ref }}$ becomes equal to $P_{m}$ at Point $\mathrm{C}$, the rotor speed converges to $\omega_{\mathrm{C}}$. Accordingly, $\omega_{\mathrm{C}}$ is higher than the minimum speed limit $\omega_{\min }$, so that this strategy effectively avoids the stalling of the wind turbine.

Period of Recovering Rotor Speed of the Wind Turbine As in (Kang et al., 2016a), if $\omega_{r}$ satisfies the following condition, $\omega_{r}$ converges.

$$
|\omega(t+T)-\omega(t)| \leq 3.45 \times 10^{-7} \text { p.u. }
$$

where $T$ is the sampling interval of $\omega_{r}$.

At Point $\mathrm{C}$, the active power $P_{\text {ref }}\left(\omega_{C}\right)$ instantly reduces $\Delta P_{s}$ to Point $C^{\prime}$ so as to recover $\omega_{r}$, and keep the value until the MPPT curve is met. At Point D, $P_{r e f}$ is switched to $P_{M P P T}$. Afterwards, $\omega_{r}$ returns from Point $\mathrm{D}$ to Point $\mathrm{A}$ by the action of the MPPT curve. $P_{r e f}$ for C-C'-D stage can be expressed as in Eq. 19.

$$
P_{r e f}=P_{r e f}\left(\omega_{C}\right)-\Delta P_{s}
$$

where $\Delta P_{s}$ is a constant.

As displayed in Figure 3B, since the condition Eq. 18 is difficult to achieve. Accordingly, this period of $\omega_{r}$ convergence
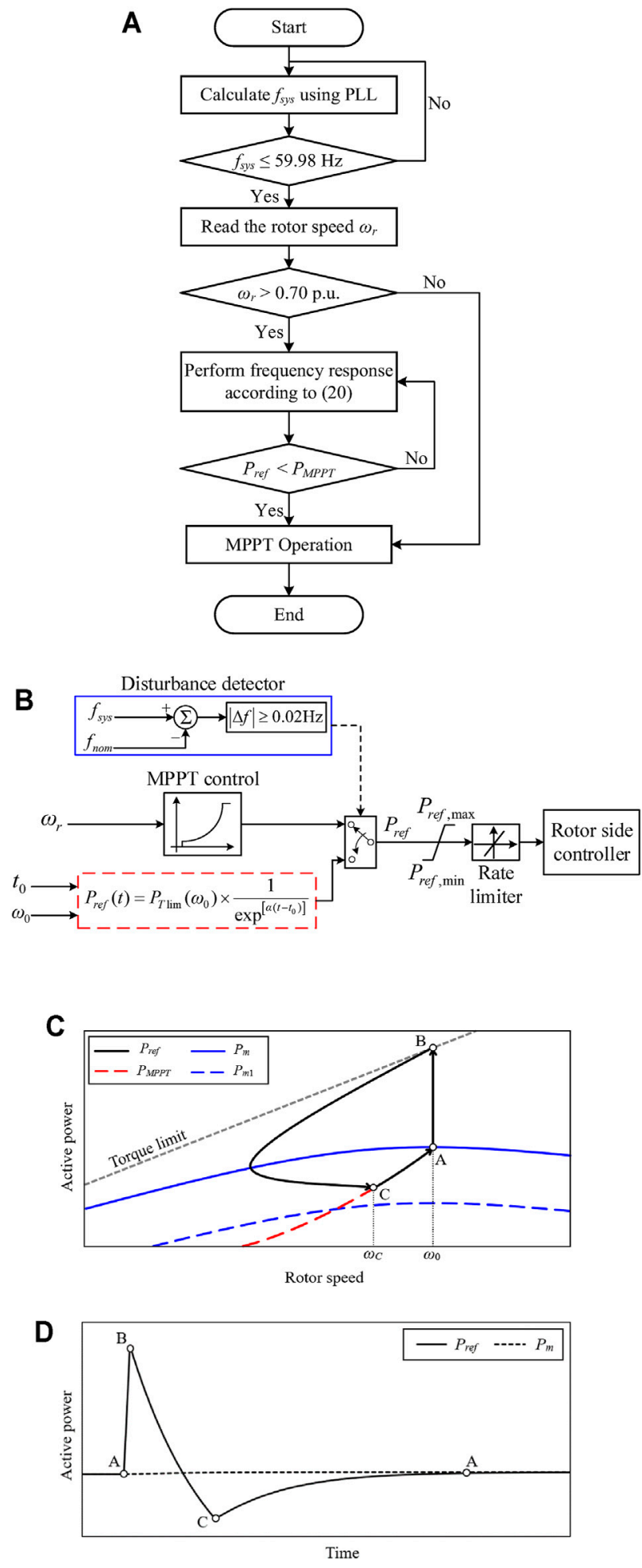

FIGURE 4 | Operational features of the proposed TLBIC scheme: (A) Flowchart of the proposed TLBIC scheme; (B) Block diagram of the proposed TLBIC scheme; (C) Power trajectory in the $\omega_{r}$ domain; (D) Active power in the time domain. 


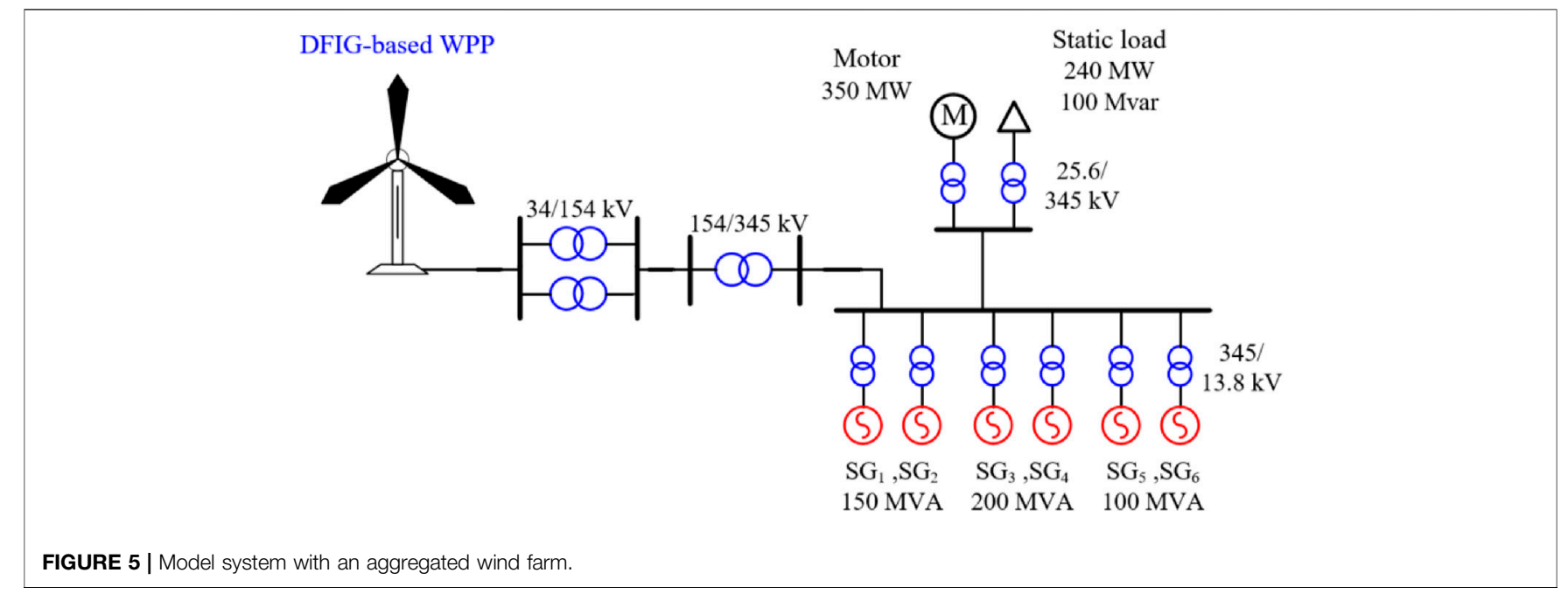

TABLE 1 | Parameters of the DFIG.

\begin{tabular}{llc} 
& Units & Values \\
\hline Inertia constant & S & 5.0 \\
Apparent power & MVA & 5.5 \\
Active power & MW & 5.0 \\
Stator voltage & kV & 2.3 \\
Stator resistance & p.u & 0.023 \\
Stator leakage reactance & p.u. & 0.18 \\
Rotor resistance & p.u. & 0.016 \\
Rotor leakage reactance & p.u. & 0.16
\end{tabular}

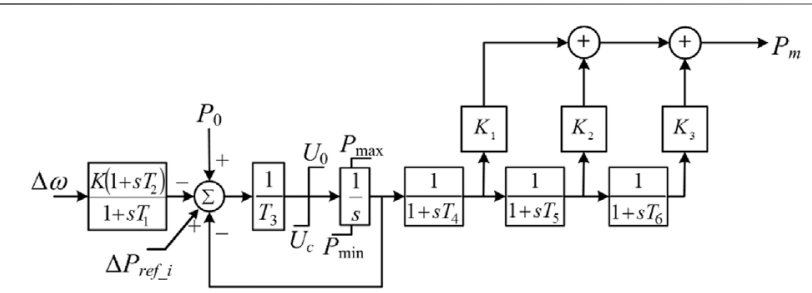

FIGURE 6 | IEEEG1 model (type B).

remains for a long time and leads to a waste of the KE which is unnecessary. In addition, to accelerate $\omega_{r}$ recovery, a large $\Delta P_{s}$ is desirable. However, too large $\Delta P_{s}$ may result in a severe SFD undoubtedly. Therefore, it is difficult to counterbalance the size of an SFD and the rotor speed recovery duration.

\section{Proposed Torque Limit-based Inertial Control Scheme of a DFIG}

To approach the above issues of the conventional TLBIC scheme, a proposed TLBIC scheme is carried out in this study.

The proposed TLBIC scheme aims to 1) improve the FN at a high level with less released kinetic energy and 2) ensure the frequency stabilization with reduced SFD. To this end, $P_{\text {ref }}$ is represented as in Eq. 20, as shown in Figure 4B.

$$
P_{r e f}(t)=P_{T \lim }\left(\omega_{0}\right) \times \frac{1}{\exp ^{\left[\alpha\left(t-t_{0}\right)\right]}} \quad t \geq t_{0}
$$

where $\alpha$ is the frequency control parameter; $t_{0}$ is the moment of disturbance occurrence.

The flowchart and the power operation features of the proposed TLBIC scheme are presented in Figures 4A,C, respectively. As the conventional TLBIC scheme, the proposed reference power increases instantly from $P_{M P P T}\left(\omega_{0}\right)$ to $P_{\text {Tlim }}\left(\omega_{0}\right)$ at $t_{0}$ (see A-B trajectory in Figure $4 \mathrm{C}$ ), so as to provide short-term frequency response. Different from the conventional reference power (which is a function of $\omega_{r}$ ), the proposed reference power is a function of the time. As time goes on, $P_{r e f}$ continues to decrease smoothly until the MPPT curve is met (see B-C trajectory in Figure 4C). At Point C, $P_{\text {ref }}$ equals $P_{m}$ and $\omega_{r}$ converges to $\omega_{C}$. Afterwards, $P_{\text {ref }}$ of the DFIG switches to $P_{M P P T}$, and the rotor speed returns to the initial state along the $P_{M P P T}$ curve for optimum power production (see C-A trajectory in Figure 4C).

Compared with the conventional TLBIC scheme, the proposed TLBIC scheme is more manageable, which can control the output active power at the initial period of the frequency response by adjusting $\alpha$. In addition, the proposed TLBIC scheme starts the restoration of $\omega_{r}$ earlier without the period of $\omega_{r}$ convergence and avoids the release of unnecessary KE. As shown in Figure 4D; Eq. 20, with the assistance of time-varying power function, $P_{\text {ref }}$ decreases smoothly with time and switches to MPPT mode without an active power mutation, which effectively ensures frequency stabilization.

It is quite important to remark that the setting of $\alpha$ in Eq. 20: a small $\alpha$ is instrumental in rapid $\omega_{r}$ restoration. However, a small $\alpha$ is not benefit to release the rotating $\mathrm{KE}$ and boost the FN; On the contrary, a large $\alpha$ could rise the FN effectively, but it delays $\omega_{r}$ recovery and causes a severe SFD. Therefore, the 


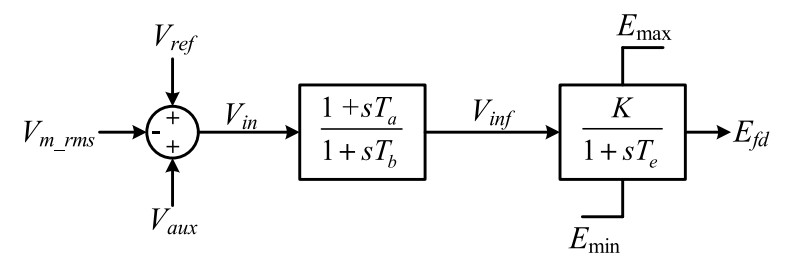

FIGURE 7 | SEXS exciter
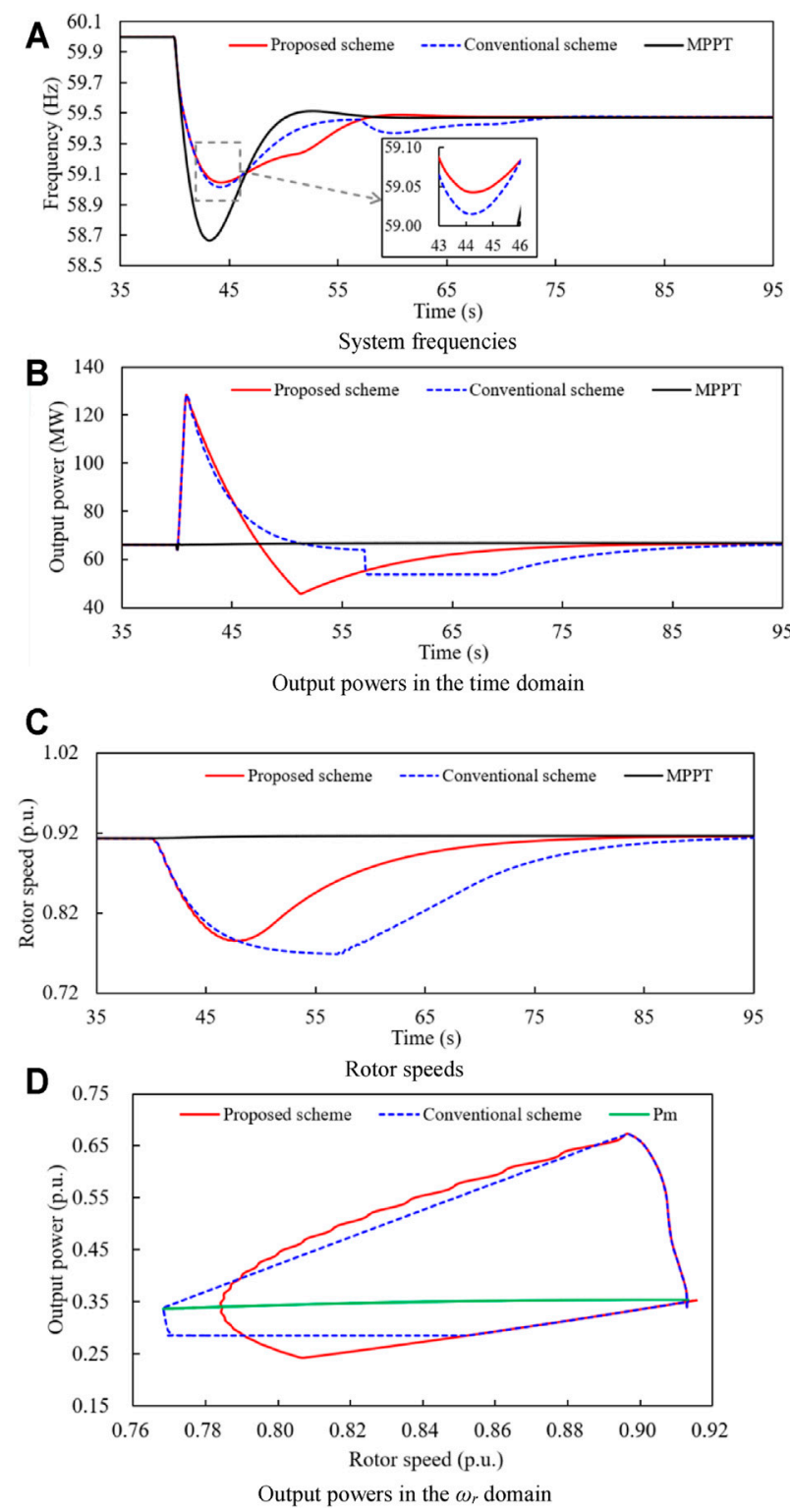

FIGURE 8 | Simulation results for case 1. (A) System frequencies. (B) Output powers in the time domain. (C) Rotor speeds. (D) Output powers in the $\omega r$ domain.

setting of $\alpha$ is critically important. Besides, $P_{\text {ref }}$ should be limited by an active power rate limiter and a maximum power limiter.

\section{MODELING OF SIMULATION SYSTEM}

Simulations on various wind power penetrations and wind speed conditions are conducted in EMTP-RV simulator to demonstrate the effectiveness of the proposed TLBIC scheme. As displayed in Figure 5, the model system consists of one aggregated DFIGbased wind farm, six synchronous generators, a 350-MW asynchronous motor, and a $240-\mathrm{MW}$ static load. The main parameters of the DFIG are shown in Table 1.

As in (Byerly et al., 1973), all synchronous generators use the IEEEG1 governor and SEXS exciter. The classical configurations of the IEEEG1 model (type B) and SEXS exciter are displayed in Figure 6, 7, respectively. Note that the secondary frequency response is not implemented in this study, thus the frequency will not eventually return to the acceptable range.

\section{CASE STUDIES}

In this section, the performances of the proposed scheme under the scenarios by varying the wind power penetration levels of 30 and $40 \%$ are verified. Moreover, the average wind speed is set to $8.0 \mathrm{~m} / \mathrm{s}$ and a stochastic wind speed model is employed in this study. As a disturbance, $\mathrm{SG}_{4}$ which generates $150 \mathrm{MW}$ prior to a disturbance is tripped at $40.0 \mathrm{~s}$.

The following several subsections will compare the performances of the proposed scheme with the conventional scheme and MPPT operation in terms of the system frequency nadir, second frequency drop, starting time of rotor speed recovery, and released kinetic energy, respectively.

The released kinetic energy $\Delta E_{r e l}$ in this paper is defined as:

$$
\Delta E_{r e l}=H_{D F I G}\left(\omega_{0}^{2}-\omega_{C}^{2}\right)
$$

\section{Case 1: Wind Power Penetration $=\mathbf{3 0} \%$ and Wind Speed $=8 \mathrm{~m} / \mathrm{s}$}

Figure 8 shows the simulation results for case 1, in which the wind power penetration level is $30 \%$ and wind speed condition is $8.0 \mathrm{~m} / \mathrm{s}$. In addition, $\Delta P_{s}$ for the conventional scheme is set to 0.05 p. u., as suggested in $(\mathrm{GB} / \mathrm{T} 19963,2011)$, and the $\mathrm{KE}$ available from the wind turbine is $1.708 \mathrm{~s}$.

Since $\mathrm{SG}_{4}$ is offline at $t=40 \mathrm{~s}$, the system active power is out-offbalanced so that the frequency drops. As shown in Figure 8A, the frequency nadirs of the proposed TLBIC scheme, conventional TLBIC scheme, and MPPT control are 59.043, 59.014, and 58.665 $\mathrm{Hz}$, respectively. When the DFIG operates at MPPT mode, it does not participate in system frequency response including inertia response and governor response, thus, its active power output and rotor speed remain unchanged. In addition, compared with the conventional strategy, the FN of the proposed TLBIC strategy is slightly boosted by $0.029 \mathrm{~Hz}$. This is mainly because $\alpha$ is set to 0.1 so that the active power of the proposed TLBIC scheme is larger in the initial period of the inertial response (Figure 8D).

Figure 8C displays the rotor speeds of case $1, \omega_{r}$ in the proposed TLBIC strategy drops faster and decreases to 0.784 p. u. at $47.6 \mathrm{~s}$, 
TABLE 2 | Simulation results for all cases.

\begin{tabular}{|c|c|c|c|c|}
\hline & & Case 1 & Case 2 & Case 3 \\
\hline \multirow[t]{3}{*}{ Frequency nadir (Hz) } & MPPT & 58.665 & 58.501 & 58.614 \\
\hline & Conventional scheme & 59.014 & 58.953 & 58.930 \\
\hline & Proposed scheme & 59.043 & 58.977 & 59.010 \\
\hline \multirow[t]{2}{*}{ Second frequency nadir $(\mathrm{Hz})$} & Conventional scheme & 59.368 & 59.265 & 59.330 \\
\hline & Proposed scheme & - & - & - \\
\hline \multirow[t]{2}{*}{ Starting time of rotor speed recovery (s) } & Conventional scheme & 57.0 & 57.0 & 57.4 \\
\hline & Proposed scheme & 47.6 & 47.6 & 47.1 \\
\hline \multirow[t]{2}{*}{ Released kinetic energy (s) } & Conventional scheme & 1.209 & 1.221 & 1.350 \\
\hline & Proposed scheme & 1.085 & 1.167 & 1.203 \\
\hline
\end{tabular}
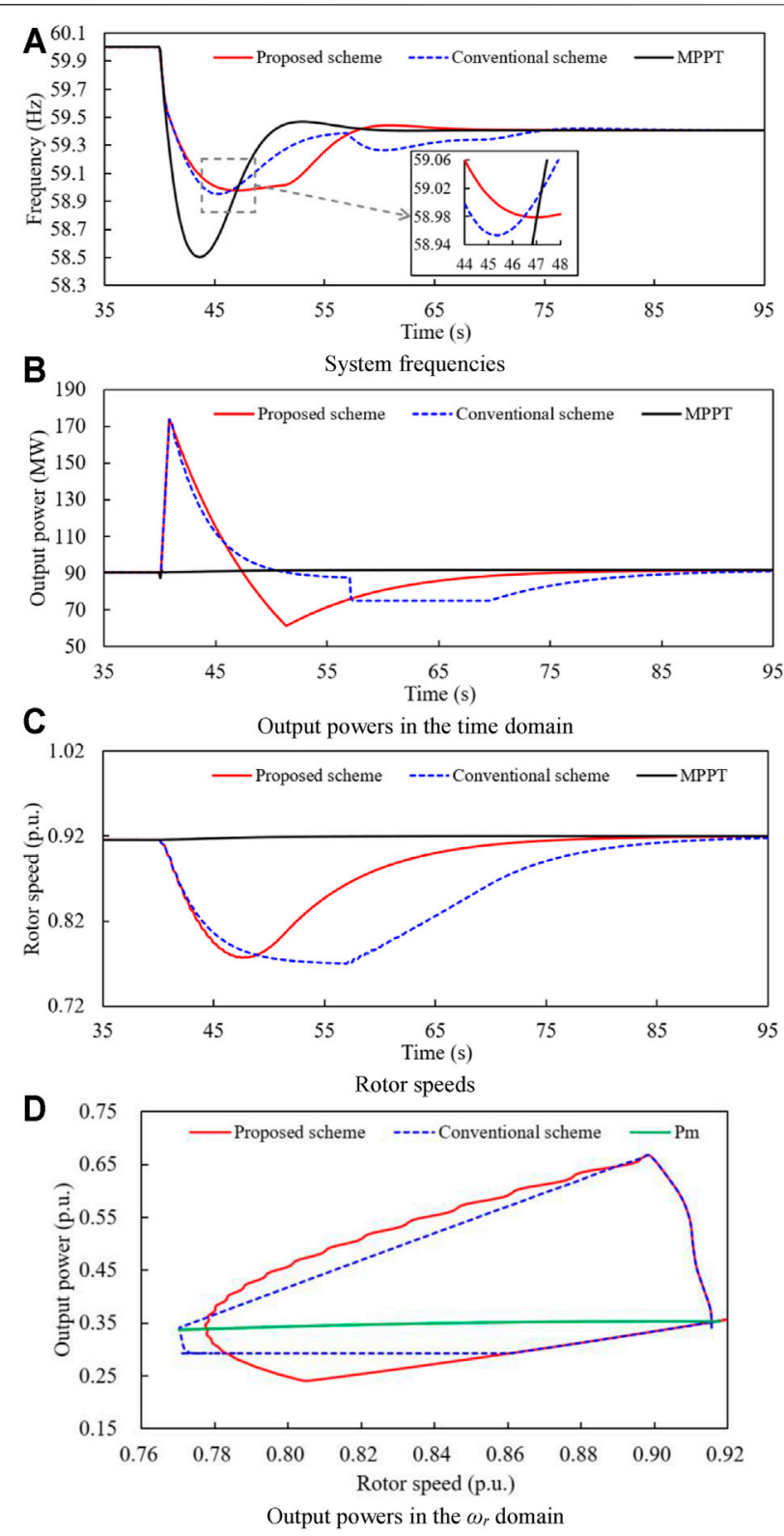

FIGURE 9 | Simulation results for case 2. (A) System frequencies. (B) Output powers in the time domain. (C) Rotor speeds. (D) Output powers in the $\omega$ r domain. and then gradually returns to $\omega_{0}$. Due to the slow rotor speed convergence, $\omega_{r}$ in the conventional TLBIC scheme converges to $0.768 \mathrm{p}$. u. at $57.0 \mathrm{~s}$. Consequently, the proposed TLBIC scheme begins the restoration of the rotor speed earlier than the conventional TLBIC scheme by $9.4 \mathrm{~s}$. Moreover, the KE released from the wind turbine during the inertial response in the proposed TLBIC scheme is $1.085 \mathrm{~s}$, while the released KE in the conventional TLBIC scheme is $1.209 \mathrm{~s}$ (Table 2). The reason for this phenomenon is that $\omega_{C}$ is more in the proposed TLBIC scheme.

In order to recover $\omega_{r}$, the conventional scheme instantly reduces the output power by $10 \mathrm{MW}$ at $57 \mathrm{~s}$ to accelerate the recovery of $\omega_{r}$, which causes a severe SFD of $0.632 \mathrm{~Hz}$ (Figures 8A,B). In contrast, the proposed reference power $P_{\text {ref }}$ decreases smoothly with time by Eq. 20, which effectively ensures the rapid rotor recovery and frequency stabilization without a fluctuation, as shown in Figure 8A.

\section{Case 2: Wind Power Penetration $=40 \%$ and Wind Speed $=8 \mathrm{~m} / \mathrm{s}$}

Figure 9 shows the simulation results for case 2, in which the wind power penetration level is more than it is in case 1 by $10 \%$.

As illustrated in Figure 9A, the FN of the MPPT operation is $58.501 \mathrm{~Hz}$, which is lower than that of case 1 by $0.164 \mathrm{~Hz}$ obviously. This mainly suffers from the higher wind power penetration. Furthermore, the frequency nadirs of the proposed and conventional TLBIC strategy are $58.977 \mathrm{~Hz}, 58.953 \mathrm{~Hz}$, respectively. Compared with the conventional TLBIC scheme, the FN of the proposed TLBIC scheme is improved by $0.024 \mathrm{~Hz}$ due to the setting of $\alpha$ in Eq. 20. In addition, the system frequencies for the proposed and conventional TLBIC strategy reach the steady state at 68.0 and $85.0 \mathrm{~s}$, respectively.

$\omega_{r}$ in the proposed and conventional TLBIC scheme start the restoration at 47.6 and $57.0 \mathrm{~s}$, respectively. Moreover, a significant SFD occurs at $60.3 \mathrm{~s}$ in the conventional TLBIC scheme while there is no frequency fluctuation in the proposed TLBIC scheme during the restoration of $\omega_{r}$. The size of the SFD for the conventional TLBIC scheme is $0.735 \mathrm{~Hz}$, which is larger than that in case 1 by $0.103 \mathrm{~Hz}$ because of a bigger $\Delta P_{s}$.

Table 2 presents the simulation results for case 2 , the released $\mathrm{KE}$ in the proposed and conventional TLBIC scheme are 1.167 and $1.221 \mathrm{~s}$, respectively. Obviously, the released $\mathrm{KE}$ of the proposed TLBIC scheme is less.

Simulation results of the above two cases clearly illustrate that the proposed TLBIC strategy can heighten the FN at a high level 

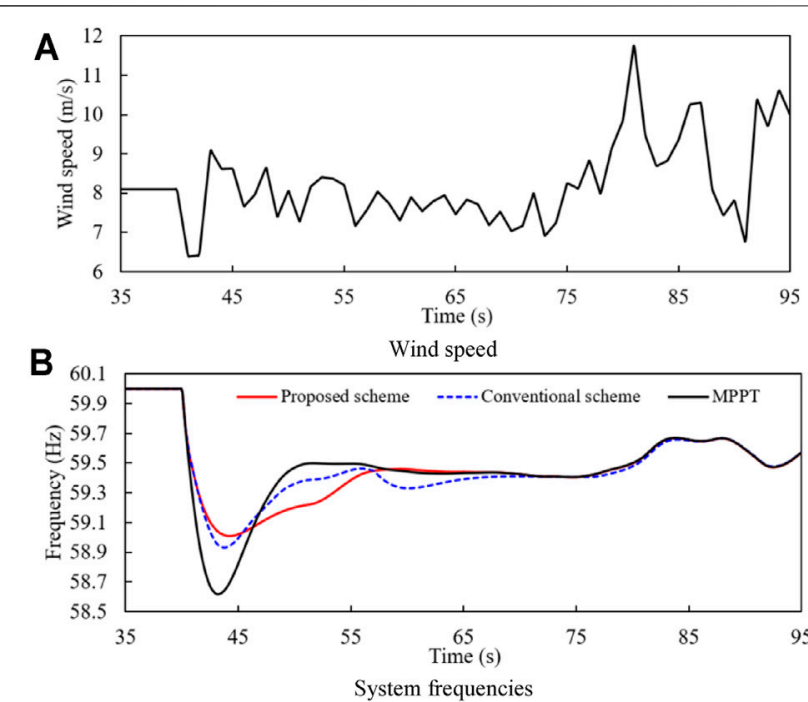

C
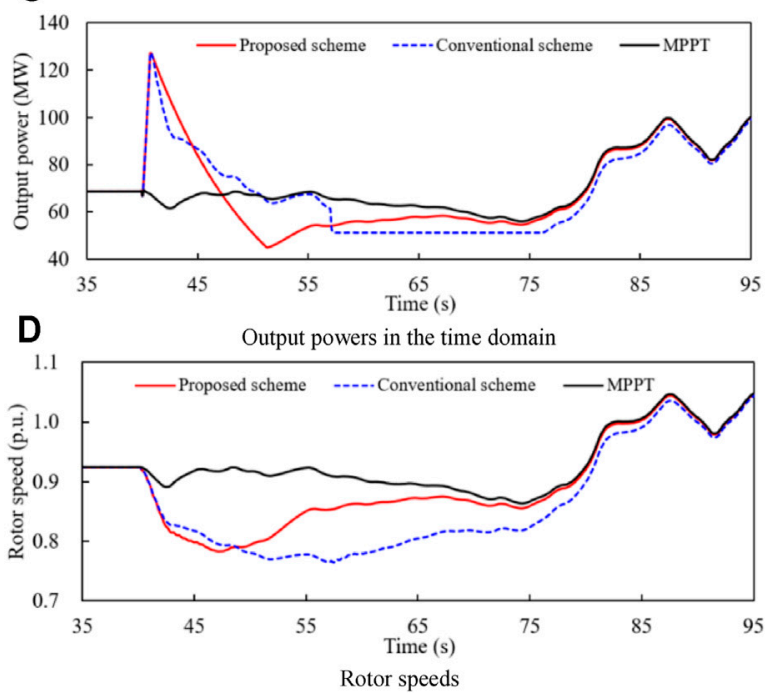

FIGURE 10 | Simulation results for case 3. (A) Wind speed. (B) System frequencies. (C) Output powers in the time domain. (D) Rotor speeds.

with less KE while guaranteeing the fast frequency stabilization under high wind power penetrations.

The above two cases have demonstrated the benefits of the proposed TLBIC strategy under constant wind speed. Whereas, considering the wind velocities are variable in a realistic scenario, the availability of the proposed TLBIC strategy will be investigated on a stochastic wind speed model in the following section.

\section{Case 3: Wind Power Penetration $=\mathbf{3 0} \%$ and Random Wind Speed Condition}

The stochastic wind speed model is presented in Figure 10A. As displayed in Figure 10B, the frequency nadirs of the proposed TLBIC scheme, conventional TLBIC scheme, and MPPT control occur at 44.2, 43.7 and $43.2 \mathrm{~s}$ and are 59.043, 59.014, and $58.665 \mathrm{~Hz}$, respectively, and the frequency nadirs of three control schemes is all lower than those of case 1 due to the influence of the wind speed drop (Figure 10A).
Moreover, the system frequencies for the proposed and conventional TLBIC scheme reach the steady state at 62.0 and $72.0 \mathrm{~s}$, respectively.

Since the DFIG output power is closely related to the wind speed, the output power raise at $73 \mathrm{~s}$ with the increase of the wind speed (Figures 10A,C). As displayed in Table 2, $\omega_{r}$ of the proposed TLBIC scheme starts the recovery at $47.1 \mathrm{~s}$ which is earlier than that of the conventional TLBIC scheme by $10.3 \mathrm{~s}$. Furthermore, the released $\mathrm{KE}$ during the frequency response in the proposed and conventional TLBIC scheme are 1.203 and $1.350 \mathrm{~s}$, respectively.

Simulation results displayed in case 3 also demonstrate that the advantages and effectiveness of the proposed TLBIC scheme. Even though in a stochastic wind speed, the proposed TLBIC scheme still heightens the FN at a high level with less KE while guaranteeing the rapid frequency stabilization with reduced SFD.

\section{CONCLUSION}

This study suggests an optimized inertial control of a DFIG for heightening the $\mathrm{FN}$ at a high level with less $\mathrm{KE}$ and guaranteeing the fast frequency stabilization under high wind power penetrations with reduced SFD. To this end, when detecting a disturbance, the active power of the DFIG increases to the torque limit so that enough rotor kinetic energy is released into the power grid, and then the power reference smoothly reduces until the MPPT curve is met. Afterwards, the rotor speed reverts to the initialization along the MPPT curve without a frequency fluctuation.

Simulation results on various wind penetrations and wind velocities significantly demonstrate that the proposed TLBIC scheme shows better performances during the frequency response over the conventional TLBIC scheme: 1) during the period of supporting the system frequency, the proposed TLBIC strategy heightens the FN at a high level with less released KE; 2) during the period of recovering the rotor speed, the proposed TLBIC scheme guarantees the rapid frequency stabilization with reduced SFD effectively. Consequently, the optimized scheme can provide an effective approach for reducing the waste of the KE and balancing the depth of the SFD and the duration of rotor speed restoration.

\section{DATA AVAILABILITY STATEMENT}

The raw data supporting the conclusions of this article will be made available by the authors, without undue reservation, to any qualified researcher. Requests to access the datasets should be directed to HW, wanghongmei@ntu.edu.cn.

\section{AUTHOR CONTRIBUTIONS}

YX contributed to the conception and design of the proposed strategy. All authors wrote and edited the manuscript.

\section{FUNDING}

This work was supported by the Natural Science Foundation of the Jiangsu Higher Education Institutions of China (20KJB470026). 


\section{REFERENCES}

Bao, W., Ding, L., and Liu, Z. (2020). Analytically derived fixed termination time for stepwise inertial control of wind turbines-Part I: Analytical derivation. Inter. J. Elect. Power Energ. Sys 121, 1-10. doi:10.1016/j.ijepes.2020.106120

Boukhezzar, B., and Siguerdidjane, H. Nonlinear control of a variable speed wind turbine using a two-mass model. IEEE Trans. Energ. Convers. 26 (1), 149-161. Mar. 2011.

Byerly, R. T., Aanstad, O., and Berry, D. H. (1973). Dynamic models for steam and hydro turbines in power system studies. IEEE Trans. Power App. Syst. 92 (6), 1904-1915.

Fernandez, L. M., Garcia, C. A., and Jurado, F. (2008). Comparative study on the performance of control systems for doubly fed induction generator (DFIG) wind turbines operating with power regulation. Energy 33 (9), 1438-1452. doi:10.1016/j.energy.2008.05.006

GB/T19963 (2011). Technical Rule for Connecting to Power System. China.

Hafiz, F., and Abdennour, A. (2015). Optimal use of kinetic energy for the inertial support from variable speed wind turbines. Renew. Energ. 80, 629-643. doi:10.1016/j.renene.2015.02.051

$\mathrm{Hu}$, Y.-L., and Wu, Y.-K. (2019). Approximation to frequency control capability of a DFIG-based wind farm using a simple linear gain droop control. IEEE Trans. Ind. Applicat. 55 (3), 2300-2309. doi:10.1109/tia.2018.2886993

Kang, M., Kim, K., and Muljadi, E. (2016). Frequency control support of a doublyfed induction generator based on the torque limit. IEEE Trans. Power Syst. 31 (6), 4574-4583. doi:10.1109/tpwrs.2015.2514240

Kang, M., Muljadi, E., Hur, K., and Kang, Y. C. (2016). Stable adaptive inertial control of a doubly-fed induction generator. IEEE Trans. Smart Grid 7 (6), 2971-2979.

Kheshti, M., Ding, L., Nayeripour, M., Wang, X., and Terzijia, V. (2019). Active power support of wind turbines for grid frequency events using a reliable power reference scheme. Renew. Energ. 139, 1421-1454. doi:10.1016/j.renene.2019.03.016

Lao, H. (2021). Innovated inertia control of DFIG with dynamic rotor speed recovery. CSEE J. Power Energ. Syst.

Lee, J., Jang, G., Muljadi, E., Blaabjerg, F., Chen, Z., and Cheol Kang, Y. (2016). Stable short-term frequency support using adaptive gains for a DFIG-based wind power plant. IEEE Trans. Energ. Convers. 31 (3), 1068-1079. doi:10.1109/ tec.2016.2532366

Li, Y., Xu, Z., and Wong, K. P. (2017). Advanced control strategies of PMSG-based wind turbines for system inertia support. IEEE Trans. Power Syst. 32 (4), 3027-3037. doi:10.1109/tpwrs.2016.2616171

Miao, L., Wen, J., Xie, H., Yue, C., and Lee, W.-J. (2015). Coordinated Control Strategy of Wind Turbine Generator and Energy Storage Equipment for Frequency Support. IEEE Trans. Ind. Applicat. 51 (4), 2732-2742. doi:10.1109/tia.2015.2394435

Morren, J., Pierik, J., and de Haan, S. W. H. (2006). Inertial response of variable speed wind turbines. Electric Power Syst. Res. 76 (11), 980-987. doi:10.1016/ j.epsr.2005.12.002

Peng, X., Yao, W., Yan, C., Wen, J., and Cheng, S. (2020). Two-Stage Variable Proportion Coefficient Based Frequency Support of Grid-Connected DFIGWTs. IEEE Trans. Power Syst. 35 (2), 962-974. doi:10.1109/tpwrs.2019.2943520

Ullah, N. R., Thiringer, T., and Karlsson, D. (2008). Temporary Primary Frequency Control Support by Variable Speed Wind Turbines- Potential and Applications. IEEE Trans. Power Syst. 23 (2), 601-612. doi:10.1109/tpwrs.2008.920076

Van de Vyver, J., De Kooning, J. D. M., Meersman, B., Vandevelde, L., and Vandoorn, T. L. (2016). Droop control as an alternative inertial response strategy for the synthetic inertia on wind turbines. IEEE Trans. Power Syst. 31 (2), 1129-1138. doi:10.1109/tpwrs.2015.2417758
Wang, S., and Tomsovic, K. (2018). A novel active power control framework for wind turbine generators to improve frequency response. IEEE Trans. Power Syst. 33 (6), 6579-6589. doi:10.1109/tpwrs.2018.2829748

Wu, Y.-K., Yang, W. H., and Hu, Y. L. Frequency regulation at a wind farm using a timing-varying inertia and droop controls. IEEE Trans. Ind. Appl. 55 (1), 213-2224. Jan./Feb. 2019.

Wu, Z., Gao, D. W., Zhang, H., Yan, S., and Wang, X. (2017). Coordinated control strategy of battery energy storage system and PMSG-WTG to enhance system frequency regulation capability. IEEE Trans. Sustain. Energ. 8 (3), 1330-1343. doi:10.1109/tste.2017.2679716

Xiong, L., Liu, L., Liu, X., and Liu, Y. (2021). Frequency Trajectory Planning Based Strategy for Improving Frequency Stability of Droop-Controlled Inverter Based Standalone Power Systems. IEEE J. Emerg. Sel. Top. Circuits Syst. 11 (1), 176-187. doi:10.1109/jetcas.2021.3052006

Xiong, L., Liu, X., Liu, Y., and Zhuo, F. (2020). Modeling and Stability Issues of Voltage-Source Converter Dominated Power Systems: a review. CSEE J. Power Energ. Syst. (Early Access), 1-18.

Xiong, L., Liu, X., Zhang, D., and Liu, Y. (2021). Rapid Power CompensationBased Frequency Response Strategy for Low-Inertia Power Systems. IEEE J. Emerg. Sel. Top. Power Electron. 9 (4), 4500-4513. doi:10.1109/ jestpe.2020.3032063

Xiong, Y., Yao, W., and Fen, J. (2021). Two-level combined control scheme of VSCHVDC integrated off shore wind farms for onshore system frequency support. IEEE Trans. Power Syst. 36 (1), 781-792.

$\mathrm{Xu}, \mathrm{G} ., \mathrm{Liu}, \mathrm{F} ., \mathrm{Hu}, \mathrm{J}$. , and Bi, T. (2018). Coordination of wind turbines and synchronous generators for system frequency control. Renew. Energ. 129, 225-236. doi:10.1016/j.renene.2018.05.104

$\mathrm{Xu}, \mathrm{G}$., and Xu, L. (2017). Improved use of WT kinetic energy for system frequency support. IET Renew. Power Generation 11 (8), 1094-1100. doi:10.1049/ietrpg.2016.0183

Yang, D., Jin, Z., Zheng, T., Jin, E., Zhang, X., and Hua, L. (2021). Frequency Control Scheme with Dynamic Droop Characteristics of a DFIG for Mitigating the Frequency Fluctuations. Int. Trans. Ele ctr. Energ. Syst. 33 (11), 1-11.

Yang, D., Jin, Z., Zheng, T., and Jin, E. (2022). An adaptive droop control strategy with smooth rotor speed recovery capability for type III wind turbine generators. Int. J. Electr. Power Energ. Syst. 135, 107532. doi:10.1016/ j.ijepes.2021.107532

Yang, D., Kim, J., Kang, Y. C., Muljadi, E., Zhang, N., Hong, J., et al. (2018). Temporary frequency support of a DFIG for high wind power penetration. IEEE Trans. Power Syst. 33 (3), 3428-3437. doi:10.1109/tpwrs.2018.2810841

Conflict of Interest: The authors declare that the research was conducted in the absence of any commercial or financial relationships that could be construed as a potential conflict of interest.

Publisher's Note: All claims expressed in this article are solely those of the authors and do not necessarily represent those of their affiliated organizations, or those of the publisher, the editors and the reviewers. Any product that may be evaluated in this article, or claim that may be made by its manufacturer, is not guaranteed or endorsed by the publisher.

Copyright (C) $2021 \mathrm{Xu}$ and Wang. This is an open-access article distributed under the terms of the Creative Commons Attribution License (CC BY). The use, distribution or reproduction in other forums is permitted, provided the original author(s) and the copyright owner(s) are credited and that the original publication in this journal is cited, in accordance with accepted academic practice. No use, distribution or reproduction is permitted which does not comply with these terms. 